\title{
ON SEQUENTIAL ESTIMATION OF A CERTAIN ESTIMABLE FUNCTION OF THE MEAN VECTOR OF A MULTIVARIATE NORMAL DISTRIBUTION
}

\author{
V. K. ROHATGI* and SURESH C. RASTOGI
}

(Received 7th July 1971)

Communicated by P. D. Finch.

Summary. Consider a $k$-variable normal distribution $\mathscr{N}(\mu, \Sigma)$ where $\mu=\left(\mu_{1}, \mu_{2}, \cdots, \mu_{k}\right)^{\prime}$ and $\Sigma$ is a diagonal matrix of unknown elements $\sigma_{i}^{2}>0, i=1,2, \cdots, k$. The problem of sequential estimation of $\Sigma_{i=1}^{k} \alpha_{i} \mu_{i}$ is considered. The stopping rule used is shown to have some interesting limiting properties when the $\sigma_{i}$ 's become infinite.

\section{Introduction}

Let $X_{1}, X_{2}, \cdots, X_{n}$ be a random sample from a $k$-variable normal population $\mathscr{N}(\mu, \Sigma)$ where $\mu=\left(\mu_{1}, \cdots, \mu_{k}\right)^{\prime}$ is the mean vector,

$$
\Sigma=\left[\begin{array}{ccccc}
\sigma_{1}^{2} & \sigma_{2}^{2} & & & 0 \\
& & & \cdot & \\
0 & & & & \sigma_{k}^{2}
\end{array}\right], \sigma_{i}^{2}<\infty, \quad i=1,2, \cdots, k
$$

is the dispersion matrix and both $\mu$ and $\Sigma$ are unknown. Sequential estimation of $\mu$ when $\Sigma$ is unknown has been considered by Khan [1] where references to previous work on the subject (for the case $k=1$ ) may also be found. We consider here the problem of estimation of

$$
v=\sum_{i=1}^{k} \alpha_{i} \mu_{i}
$$

where $\alpha_{i}, i=1,2, \cdots, k$ are given real numbers. We assume, without loss of generality, that $\alpha_{i} \neq 0, i=1,2, \cdots, k$. Let

$$
X_{i n}=n^{-1} \sum_{j=1}^{n} X_{i j}, S_{i n}^{2}=(n-1)^{-1} \sum_{j=1}^{n}\left(X_{i j}-X_{i n}\right)^{2},
$$

* Supported by the NSF Grant Number GP-22585. 
where $n \geqq 2$ and $X_{i}=\left(X_{i 1}, \cdots, X_{i n}\right)^{\prime}, i=1,2, \cdots, \kappa$. Let $\bar{X}_{n}=\left(X_{1 n}, \cdots, \not_{k n}\right)^{\prime}$ Following [5] we use $Y=\sum_{i=1}^{k} \alpha_{i} \bar{X}_{i n}$ as an unbiased estimate of $v$ and measure the loss incurred by

$$
L(n)=|Y-\nu|^{s}+n
$$

where $s>0$ is a given real number. Clearly

$$
\phi(n)=E L(n)=a(s)\left(\Sigma_{1}^{\kappa} \alpha_{i}^{2} \sigma_{i}^{2}\right)^{s / 2} n^{-s / 2}+n,
$$

where $a(s)=(\sqrt{2 \pi})^{-1} 2^{(s+1) / 2} \Gamma((s+1) / 2)$. The risk $\phi(n)$ is minimized for $n=n_{0}$ given by

$$
n_{0}=\beta^{2 /(s+2)}\left(\Sigma \alpha_{i}^{2} \sigma_{i}^{2}\right)^{s /(s+2)}
$$

where $\beta=s a(s) / 2$. If $\sigma_{i}$ 's are known we take $n_{0}$ observations and estimate $v$ by $Y$. The risk in doing so is given by

$$
v(\sigma)=\phi\left(n_{0}\right)=(1+2 / s) n_{0} .
$$

Since $\sigma=\left(\sigma_{1}, \cdots, \sigma_{k}\right)^{\prime}$ is not known we determine a sample size $N$ by means of the following sequential procedure.

Let

$$
\left\{\begin{array}{l}
N=\text { smallest integer } n \geqq m \text { for which } \\
n>\left(\beta^{2 / s} \Sigma_{1}^{k} \alpha_{i}^{2} S_{i n}^{2}\right)^{s /(s+2)}
\end{array}\right.
$$

where $m$ is the starting sample size.

\section{Results}

In what follows we write $\sigma \rightarrow \infty$ to mean that $\sigma_{i} \rightarrow \infty, i=1,2, \cdots, k, c$ will denote a generic positive constant.

Write

$$
\begin{aligned}
\sigma_{*}=\min \left(\sigma_{1}, \sigma_{2}, \cdots, \sigma_{k}\right), & \sigma^{*}=\max \left(\sigma_{1}, \sigma_{2}, \cdots, \sigma_{k}\right), \\
\alpha_{*}^{2}=\min \left(\alpha_{1}^{2}, \alpha_{2}^{2}, \cdots, \alpha_{k}^{2}\right), & \alpha^{*^{2}}=\max \left(\alpha_{2}^{2}, \alpha_{2}^{2}, \cdots, \alpha_{k}^{2}\right),
\end{aligned}
$$

and assume that

$$
\sigma^{*} / \sigma_{*} \rightarrow 1 \text { as } \sigma \rightarrow \infty \text {. }
$$

Note that

$$
\beta^{2 /(2+s)}\left(k \alpha_{*}^{2} \sigma_{*}^{2}\right)^{s /(s+2)} \leqq n_{0} \leqq \beta^{2 /(s+2)}\left(k \alpha^{*^{2}} \sigma^{* 2}\right)^{s /(s+2)}
$$

Some immediate results follow along the lines of [1].

Lemma 1. $P\{N>\infty\}=1$. 
THEOREM 1. (i) $\lim _{\sigma \rightarrow \infty} n_{0}^{-1} N=1$ a.s.

(ii) $\lim _{0 \rightarrow \infty} \mathscr{E}\left(n_{0}^{-1} N\right)=1$

We remark that both Lemma 1 and Theorem 1 hold if we replace the loss function (1) by

$$
L^{*}(n)=|Y-v|^{s}+\log n .
$$

Lemma 2. $P\{N=m\}=O\left(\sigma_{*}^{-k(m-1)}\right)$ as $\sigma \rightarrow \infty$ and (8) holds.

Lemma 3. For fixed $\theta, 0<\theta<1$

$$
P\left\{N \leqq \theta n_{0}\right\}=O\left(\sigma^{*-k(m-1)}\right) \text { as } \sigma \rightarrow \infty \text { and (8) holds. }
$$

The proofs of Lemmas 2 and 3 can easily be constructed by minor modification of the methods of Simons [4]. For example, in case of Lemma 2, we have

$$
\begin{aligned}
P\{N=m\} & =P\left\{\Sigma_{1}^{k} \alpha_{i}^{2} S_{i m}^{2} \leqq \beta^{-2 / s} m^{(s+2) / s}\right\} \\
& \leqq P\left\{\Sigma_{1}^{k} \frac{(m-1) S_{i m}^{2}}{\sigma_{i}^{2}} \leqq \beta^{-2 / s} \sigma_{*}^{-2} \alpha_{*}^{-2}(m-1) m^{(s+2) / s}\right\} \\
& =P\left\{\gamma_{k(m-1)}^{2} \leqq \beta^{-2 / s} \sigma_{*}^{-2} \alpha_{*}^{-2}(m-1) m^{(s+2) / s}\right\} \\
& =O\left(\sigma_{*}^{-k(m-1)}\right) \text { as } \sigma \rightarrow \infty
\end{aligned}
$$

Again

$$
\begin{aligned}
P\{N=m\} & \geqq P\left\{\bigcap_{i=1}^{k}\left[\alpha_{i}^{2} S_{i m}^{2} \leqq \beta^{-2 / s} k^{-1} m^{(s+2) / s}\right]\right\} \\
& \geqq\left[P\left\{\chi_{m-1}^{2} \leqq \beta^{-2 / s} k^{-1} \sigma^{*-2} \alpha^{*-2}(m-1) m^{(s+2) / s}\right\}\right]^{k} \\
& =O\left(\sigma^{*-k(m-1)}\right) \text { as } \sigma \rightarrow \infty .
\end{aligned}
$$

This completes the proof of Lemma 2. The proof of Lemma 3 can be constructed in a similar manner.

Theorem 2. As $\sigma \rightarrow \infty$ and (8) holds

$$
\mathscr{E} L(N)-v(\sigma)=O(1)
$$

if and only if $m \geqq s / k+1$.

PROOF. For the proof we will follow the analysis of [5] closely and indicate only the modifications required, First, using an argument similar to the one used in Lemma 3 of [2] we have $\mathscr{E}\{L(N) \mid N=n\}=\mathscr{E} L(n)$ so that

$$
\bar{v}(\sigma)=\mathscr{E} L(N)=\frac{2}{s} n_{0}^{(s+2) / 2} \mathscr{E} N^{-s / 2}+\mathscr{E} N .
$$


As in [5] we write $w(\sigma)=\tilde{v}(\sigma)-v(\sigma)$ for the regret and see that

$$
w(\sigma)=\frac{2}{s} n_{0}^{(s+2, / 2} \mathscr{E}\left\{N^{-s / 2}-n_{0}^{-s / 2}\right\}+\mathscr{E}\left\{N-n_{0}\right\}
$$

The necessity part of the proof is obtained by simply replacing $O\left(\sigma^{-(m+1)}\right)$ by $O\left(\sigma_{*}^{-:(m-1)}\right)$ in the computations on page 287 of [5].

For the sufficiency part we obtain, as in [5],

$$
w(\sigma) \leqq O\left(\sigma^{* s}\right)\left[O\left(\sigma_{*}^{-k(m-1)}\right)+O\left(\sigma_{*}^{-}\right) \mathscr{E}\left\{\frac{\left(N-n_{0}\right)^{2}}{n_{0}}\right\}\right]
$$

and it suffices to show that

$$
\mathscr{E}\left\{\frac{\left(N-n_{0}\right)^{2}}{n_{0}}\right\}=O(1)
$$

as $\sigma \rightarrow \infty$ and (8) holds. On integration by parts one obtains

$$
\begin{aligned}
\mathscr{E}\left\{\frac{\left(N-n_{0}\right)^{2}}{n_{0}}\right\} \leqq 1+2 \int_{1}^{\sqrt{n_{0}}} \lambda P\left\{N-n_{0}<-\lambda \sqrt{n_{0}}\right\} d \lambda \\
+2 \int_{1}^{\infty} \lambda P\left\{N-n_{0}>\sqrt{n_{0}}\right\} d \lambda,
\end{aligned}
$$

which is inequality (11) in [5]. We have

$$
\begin{aligned}
& 2 \int_{1}^{\sqrt{n_{0}}} \lambda P\left\{N-n_{0}<-\lambda \sqrt{n_{0}}\right\} d \lambda \\
& \leqq n_{0} P\left\{N \leqq \frac{1}{2} n_{0}\right\}+2 \int_{1}^{\sqrt{n_{0}} 2} \lambda P\left\{N-n_{0}<-\lambda \sqrt{n_{0}}\right\} d \lambda \\
& \leqq O(1)+2 \int_{1}^{\sqrt{n_{\mathrm{o}}} / 2} \lambda P\left\{\Sigma_{1}^{k} \alpha_{i}^{2} S_{i l}^{2}<\beta^{-2 / s}\left(n_{0}-\lambda \sqrt{n_{0}}\right)^{(s+2 / *} ; l \geqq n_{0} / 2\right\} d \lambda \\
& =O(1)+2 \int_{1}^{\infty} \lambda P\left\{\Sigma_{1}^{k} \alpha_{i}^{2}\left(S_{i l}^{2}-\sigma_{i}^{2}\right)<\beta^{-2 / s}\right. \\
& \left.\left[\left(n_{0}-\lambda \sqrt{n_{0}}\right)^{(s+2) / s}-n_{0}^{(s+2) / 2}\right] ; \quad l \geqq n_{0} / 2\right\} d \lambda \\
& \leqq O(1)+2 \int_{1}^{\infty} \lambda \Sigma_{1}^{k} P\left\{\alpha_{i}^{2}\left(S_{. l}^{2}-\sigma_{i}^{2}\right)\right. \\
& \left.<-\beta^{-2 / s} k^{-1}(s+2) s^{-1}\left(n_{0} / 2\right)^{2 / s} \lambda \sqrt{n_{0}} ; l>n_{0} / 2\right\} d \lambda \\
& =O(1)+2 \Sigma_{1}^{k} \int_{1}^{\infty} \lambda\left[\frac{\alpha_{i}^{2} k \beta^{2 / s}(s+2)}{2 \lambda}\right]^{4} n_{0}^{-(8+2 s) / s} \mathscr{E}\left|S_{i n_{2}}^{2}-\sigma_{i}^{2}\right|^{4} d \lambda
\end{aligned}
$$

where $n_{2}$ is the greatest integer $\leqq n_{0} / 2$ and we have used exactly the same argument as in [5] page 288 . 
Since

it follows that

$$
\begin{aligned}
n_{0}^{-(8+2 s) / *} \mathscr{E}\left|S_{i n_{2}}^{2}-\sigma_{i}^{2}\right|^{4} & \leqq c n_{0}^{-(8+2 s) / s} n_{2}^{-2} \sigma_{i}^{8} \\
& \leqq c n_{0}^{-(8+4 s) /:} \sigma^{* 8}=O(1),
\end{aligned}
$$

$$
2 \int_{1}^{\sqrt{n_{\mathrm{o}}}} \lambda P\left\{N-n_{0}<-\lambda \sqrt{n_{0}}\right\} d \lambda=0(1) .
$$

A similar argument can be used to show the boundedness of the second integral in (14). This completes the proof of the theorem.

Finally we remark that Rohatgi and O'Neill [3] have established recently that the risk is bounded if one uses the sequential procedure described by Khan [1] to estimate the mean vector of a multivariate normal population.

\section{References}

[1] R. A. Khan, 'Sequential estimation of the mean vector of a multivariate normal distribution'. Sankyhä, 30, Ser. A (1968), 331-334.

[2] H. Robbins, Sequential estimation of the mean of a normal population.Probability and Statistics, (John Wiley \& Sons, N. Y.) (1959), 235-245.

[3] V. K. Rohatgi, and R. T. O'Neill, 'On the sequential estimation of the mean vector of a multionormal population' (To appear in Ann. Inst. Statist. Maths.)

[4] G. Simons, 'On the cost of not knowing the variance when making a fixed width confidence interval for the mean'. Ann. Math. Stat. 39 (1968), 1946-1952.

[5] N. Starr, and M. B. Woodroofe, 'Remarks on sequential point estimation', Proc. National Acad. Sciences, 63 (1969), 285-288.

\section{Statistical Laboratory}

Catholic University of America and University of Maryland

Washington D. C. 20017 U. S. A.

\section{Present address}

Bowling Green State University

Bowling Green, Ohio, U.S.A. 